\title{
Recent Advancements in Hematology: Knowledge, Methods and Dissemination, Part 1
}

\author{
Jude Fitzgibbon ${ }^{1}$, Sophie Park ${ }^{2}$, Gordon Cook ${ }^{3}{ }^{(0)}$, Bruno Paiva $\left.{ }^{4}{ }^{(}\right)$, Annunziata Gloghini ${ }^{5}(\mathbb{D}$, \\ Eric Van Breda ${ }^{6}$, Fabiana Busti ${ }^{7}{ }^{\mathbb{D}}$, Laurent Garderet ${ }^{8}$, Ricardo Dolcetti ${ }^{9}{ }^{\circledR}$, Marie Robin ${ }^{10}{ }^{(0}$, \\ Rodrigo Martino ${ }^{11}$, Alessandro Busca ${ }^{12}$, Michèle Sabbah ${ }^{13}\left(0\right.$, Salvatore De Rosa ${ }^{14}(\mathbb{D}$, \\ Maurizio Martini ${ }^{15}{ }^{(0)}$, Francesco Onida ${ }^{16}{ }^{\circ}$, Pierre Aucouturier ${ }^{17}$, Fredrik Schjesvold ${ }^{18,19}{ }^{(0)}$, \\ Stéphane Minvielle ${ }^{20,21}{ }^{\circ}$, Mario Mazzucato ${ }^{22} \oplus$, Carolina Terragna ${ }^{23}$, Michel Delforge ${ }^{24}$, \\ Claire Harrison ${ }^{25}$ and Antonino Carbone $26, * \mathbb{B}$
}

1 Centre for Haemato-oncology, Barts Cancer Institute, QMUL, London EC1M 6B, UK; j.fitzgibbon@qmul.ac.uk

2 Clinique Universitaire d'Hématologie, Grenoble Alpes University Hospital, Institute for Advanced Biosciences, Grenoble Alpes University, INSERM U1209, CNRS UMR 5309, CS 10217, 38043 Grenoble CEDEX 09, France; spark@chu-grenoble.fr

3 Section of Experimental Haematology, Leeds Institute of Cancer \& Pathology, University of Leeds, St James's University Hospital, Beckett Street, Leeds LS9 7TF, UK; G.Cook@Leeds.ac.uk

4 Instituto de Investigacion Sanitaria de Navarra (IDISNA), Centro de Investigacion Medica Aplicada (CIMA), Clinica Universidad de Navarra, CIBER-ONC number CB16/12/00369, 31008 Pamplona, Spain; Bpaiva@unav.es

5 Department of Diagnostic Pathology and Laboratory Medicine, Fondazione IRCCS, Istituto Nazionale dei Tumori di Milano, Via G. Venezian 1, I-20133 Milano, Italy; annunziata.gloghini@istitutotumori.mi.it

6 Department of Rehabilitation Sciences \& Physiotherapy, Antwerp Multidisciplinary Metabolic Research Unit (AM2RUN), University of Antwerp, B-2000 Antwerp, Belgium; eric.vanbreda@uantwerpen.be

7 Department of Medicine, Section of Internal Medicine, University of Verona, Piazzale L.A. Scuro, 10, 37134 Verona, Italy; fabiana.busti@univr.it

8 Service d'Hématologie, Hôpital Pitié Salpêtrière, 47-83 boulevard de 1'hôpital, F-75013 Paris, France; laurent.garderet@aphp.fr

9 The University of Queensland Diamantina Institute, Translational Research Institute, 37 Kent Street Woolloongabba, QLD 4102, Australia; r.dolcetti@uq.edu.au

10 Hôpital Saint-Louis, APHP, INSERM 1131 Université de Paris, Paris, France; marie.robin@aphp.fr

11 Divison of Clinical Hematology, Hospital de la Santa Creu i Sant Pau, 08041 Barcelona, Spain; rmartino@santpau.cat

12 Azienda Ospedaliera-Universitaria Città della Salute e della Scienza di Torino, 10126 Turin, Italy; abusca@cittadellasalute.to.it

13 Sorbonne Université, INSERM U938, Centre de Recherche Saint-Antoine, F-75012 Paris, France; michele.sabbah@inserm.fr

14 Department of Medical \& Surgical Sciences, Magna Graecia University of Catanzaro, I-88100 Catanzaro, Italy; saderosa@unicz.it

15 Institute of Pathology, Catholic University of Sacred Heart, Largo A. Gemelli 8, 00168 Rome, Italy; maurizio.martini@unicatt.it

16 Fondazione IRCCS Ca' Granda Ospedale Maggiore Policlinico, University of Milan, 20122 Milan, Italy; francesco.onida@unimi.it

17 Department of Immunology, Inserm UMRS 938, Saint Antoine Hospital; Sorbonne Université, F-75012 Paris, France; pierre.aucouturier@inserm.fr

18 Oslo Myeloma Center, Oslo University Hospital, 0424 Oslo, Norway; fredrikschjesvold@gmail.com

19 KG Jebsen Center for B Cell Malignancies, University of Oslo, 0424 Oslo, Norway

20 Université de Nantes, CNRS, INSERM, CRCINA, F-44000 Nantes, France; stephane.minvielle@univ-nantes.fr

21 CHU de Nantes, F-44000 Nantes, France

22 Centro di Riferimento Oncologico di Aviano (CRO) IRCCS Aviano, via F.Gallini n.2, 33081 Aviano (PN), Italy; mmazzucato@cro.it

23 Institute of Hematology “L. e A. Seràgnoli”, Azienda Ospedaliera Sant'Orola- Malpighi, 40138 Bologna, Italy; carolina.terragna@unibo.it 
24 Department of Hematology, University Hospital Leuven, B-3000 Leuven, Belgium; michel.delforge@uzleuven.be

25 Department of Hematology, Guy's and St. Thomas' NHS Foundation Trust, London SE1 9RT, UK; Claire.Harrison@gstt.nhs.uk

26 Centro di Riferimento Oncologico di Aviano (CRO), IRCCS, Via F. Gallini 2, I-33081 Aviano, Italy

* Correspondence: acarbone@cro.it

Received: 23 June 2020; Accepted: 1 July 2020; Published: 1 July 2020

\section{Introduction}

Recent Advancements in Hematology: Knowledge, Methods and Dissemination is a series of editorials which is published on a biannual basis by the editorial board of the journal Bloods. In these editorials, we highlight in brief reports (of about one hundred words) a number of recently published articles that describe the most recent advancements in hematology. In this way, we hope Bloods will not only publish high-level scientific articles, but will also discuss their significance in the scientific scenario, which includes the transfer of information to the media.

\section{DLBCL: Divide and Conquer}

Highlighted by Jude Fitzgibbon

Wright et al. [1] build on their previous studies [2] with the development of the LymphGen algorithm, which combines mutation, copy number, and fusion status, to assign $>60 \%$ of Diffuse Large B Cell Lymphoma (DLBCL) tumors to seven subtypes, all having distinctive molecular features and clinical outcomes. The profile of each subtype reveals novel observations on cell of origin, dysregulated pathways, and composition of the immune microenvironment, and proposes routes to histologic transformation of indolent lymphomas. CRISPR loss-of-function screens uncovered therapeutic vulnerabilities of each subtype, including the dependence of BN2 tumors on BCR-NFKB signaling. It is encouraging that these molecular subtypes largely converge on groups defined by other large-scale initiatives $[3,4]$, despite differing patient series, sequencing approaches, and statistical methodologies. The emerging consensus lends confidence to testing these new classifications prospectively in biomarker-directed clinical trials.

\section{Benefits of Iron Chelation in MDS Patients: The TELESTO Trial}

Highlighted by Sophie Park

Myelodysplastic syndromes (MDS) are clonal hematopoietic stem cell disorders characterized by cytopenias and progression to acute myeloid leukemia (AML). Although several treatments for MDS are available, the mainstay of therapy for most patients remains supportive care. This includes red blood cell (RBC) transfusion to correct anemia, which leads to iron overload. RBC transfusion dependence and iron overload portend inferior overall survival. Some studies indicate that iron chelation therapy (ICT) may have beneficial effects on clinical endpoints in MDS; however, these data are from non-randomized trials and the validity of the results is vigorously debated.

TELESTO is the first prospective randomized double-blind placebo-controlled study testing the impact of ICT with deferasirox on a composite endpoint; e.g., event-free survival including non-fatal events such as echocardiographic evidence of worsening cardiac function, hospitalization for congestive heart failure, liver function impairment, cirrhosis, and transformation to acute myeloid leukemia [5]. It shows that median EFS with DFX is improved by one year with an HR 0.64, supporting the clinical benefit of ICT with deferasirox in low-risk and intermediate-1-risk MDS. This study is important as it is the first and the last prospective randomized trial demonstrating this advantage after a decade of retrospective non-controlled studies. 


\title{
4. Improving Early Infection-Related Morbidity and Mortality May Allow More Effective Delivery of Systemic Anti-Cancer Therapy (SACT) in Multiple Myeloma (MM)
}

\author{
Highlighted by Gordon Cook
}

Myeloma survival has substantially improved; a substantial proportion of early deaths, and deaths in remission, are due to infections. Infections induce considerable morbidity and frequently lead to SACT interruption or discontinuation, resulting in inferior treatment responses with poorer survival. Susceptibility to infection is primarily the consequence of acquired tumor-related deficiency of both humoral and cellular immunity. The publication by Drayson et al. [6] in Lancet Oncology is a welcomed focus on supportive care in myeloma. They present the findings that showed that 12 weeks of fixed-duration levofloxacin prophylaxis reduced the occurrence of febrile episodes and deaths with a hazard ratio [HR] 0.66, $(95 \%$ CI $0.51-0.86 ; p=0.0018)$ when levofloxacin was delivered prophylactically compared to placebo. Independent to levofloxacin, use of prophylactic low dose co-trimoxazole significantly reduced febrile infections and death, though this was not protocol-driven, rather as a consequence of local standard of care. Additionally, use of levofloxacin for a fixed duration was not associated with an increase in adverse events, especially healthcare-associated infections such as $C$. difficile and MRSA. The results of this trial provide a good basis for considering fixed-duration quinolone prophylaxis for newly diagnosed patients with MM undergoing SACT.

\section{Genetic Versus Immune Evolution behind Myeloma Progression}

\section{Highlighted by Bruno Paiva}

Using single-cell RNA sequencing of bone marrow cells from the precursor stages of monoclonal gammopathy of unknown significance and smoldering multiple myeloma (SMM), to full-blown MM alongside healthy donors, Zavidij and colleagues [7] confirmed previous concepts about immune changes during patient progression [8], and provided unprecedented resolution about the immune microenvironment in precursor and malignant stages of MM. Interestingly, the same group of authors reported recently that most genetic alterations present in MM patients have already occurred by the time of SMM diagnosis, some of which predict risk of progression [9]. Taken together, both studies support the relevance of genetic and immune evolution behind the progression of MM. However, while the core of genetic abnormalities remains relatively stable over time, there seems to be profound editing of the tumor-immune crosstalk. These observations provide rational therapeutic approaches aiming at enhance or restoring patients' immune status to prevent malignant transformation of SMM patients at greater risk of progression.

\section{A Biologic Agent is Involved in the Pathogenesis of a Subset of NLPHL}

\section{Highlighted by Annunziata Gloghini}

In the study by Thurner and colleagues, "Lymphocyte predominant cells detect Moraxella catarrhalis-derived antigens in nodular lymphocyte-predominant Hodgkin lymphoma" [10], the authors provide evidence that chronic antigenic stimulation by Moraxella catarrhalis (M. catarrhalis) contributes to lymphomagenesis in immunoglobulin $\mathrm{D}(\mathrm{IgD})$ positive nodular lymphocyte predominant Hodgkin lymphoma (NLPHL). Moraxella catarrhalis is a common bacterium colonizing the upper respiratory tract that express a $200 \mathrm{kDa}$ IgD-binding protein (MID/hag), enabling M. catarrhalis to bind to the $\mathrm{Fc}$ part of $\operatorname{IgD}$ and thus activating $\operatorname{IgD}$-carrying B-cells. $\operatorname{IgD}$ is expressed in the lymphocyte predominant (LP) cells in about $25 \%$ of NLPHL cases [11].

Thurner and colleagues suggest that the persistent or recurrent presence of $M$. catarrhalis presumably induces a germinal center (GC) reaction resulting in production of class-switched anti-DNA-directed RNA polymerase sub-unit beta'(RpoC) serum antibodies. The costimulatory effect of MID/hag selects for retention of IgD on a fraction of the stimulated GC B cells. Meanwhile a clone acquires genetic alterations and may transform into LP cells. However, the transformed IgD 
+ LP cells still gain growth advantage by the interaction of their BCRs with M. catarrhalis RpoC and MID/hag.

Anti-M. catarrhalis therapies should be assessed and validation of morphological and phenotypic surrogates encouraged to identify NLPHL cases related to M. catarrhalis.

\section{7. "Into Thin Air": Living High or Staying Low}

Highlighted by Eric van Breda

Thirty-five (35) years ago Stray-Gundersen et al. [12] proposed an alternative training method for elite athletes to boost their endurance capacity. Living at $2500 \mathrm{~m}$ above sea level (masl) for 27 days with regular training sessions at sea level increased performance significantly through, among other things, increases in hemoglobin concentration. To date, professional endurance athletes still make use of this altitude training to boost the oxygen-carrying capacity to working muscles for performance increments.

Interestingly, Staub et al. recently showed that adaptation to altitude is far more subtle than previously thought [13]. In contrast to the general assumption that adaptation to altitude only occurs above 2500 masl, Staub et al. found increases in hemoglobin concentration and thus oxygen carrying capacity from 300 masl onwards in Swiss men.

Although more research is needed, the important finding of the study of Staub et al. is that adaptation to thin air occurs at lower altitudes than expected and maybe altitude training camps for elite athletes should be viewed from a different perspective.

\section{Iron Homeostasis and Viral Pulmonary Infections: Possible Insights from COVID-19}

\section{Highlighted by Fabiana Busti}

Iron is essential for many biological functions, including immunity. During infections, hepcidin-mediated iron redistribution causes hypoferremia. It represents a defense mechanism against invading pathogens, such as viruses, which need the iron-dependent machinery of the host cells to replicate and spread efficiently in the body [14]. However, hypoferremia can become harmful for the immune cells, and it has been associated with poor outcomes in critically ill patients [15].

Although high ferritin levels appeared an independent predictor of mortality [16], the role of iron homeostasis in COVID-19 patients has remained largely unexplored until now.

In this study, Shah et al. have investigated the iron parameters in a small group of COVID-19 patients admitted to the intensive care unit [17]. Lower serum iron levels were associated with more severe hypoxemic respiratory failure (defined as $\mathrm{PaO}_{2} / \mathrm{FiO}_{2}$ ratio $<100$ ) and lymphopenia, irrespective of the inflammation extent, suggesting a possible role for hypoferremia as an early biomarker of disease severity.

This study provides interesting insights to understand the implications of iron dysregulation not only in COVID-19 patients but more generally in subjects with viral respiratory infections and lung damage.

\section{Adding a Monoclonal Antibody to Treat Newly Diagnosed Transplant Ineligible Myeloma Patients Improves Overall Survival}

\section{Highlighted by Laurent Garderet}

There are currently three ways to treat first line non-autologous stem-cell transplantation eligible myeloma patients: either the combination of an alkylating agent, melphalan, plus steroid and a proteasome inhibitor, bortezomib, the so-called VMP combination or the combination of an immunomodulatory agent, lenalidomide plus steroid (Rd) and more recently adding bortezomib to $\mathrm{Rd}$ (VRd) [18]. MV Mateos et al. [19], in a randomized phase 3 trial, added the anti-CD38 monoclonal antibody daratumumab [20] to VMP (D-VMP) until disease progression. Compared to VMP fixed duration, it improved not only the PFS but also the OS with $40 \%$ reduction in the risk of death and reached an approximately four-fold higher rate of negative status for minimal residual disease. The 
additional toxicity was mostly grade $1-2$ respiratory infections. This is a major achievement but it is already challenged by the addition of daratumumab to RD [21] or VRd [22]. However, it definitely demonstrates that myeloma patients should receive a monoclonal antibody to most, if not any, drug combinations in the first line setting, similarly to what has been largely proved in the relapse setting. $\mathrm{D}-\mathrm{VMP}$ is a new standard-of-care for these patients but is challenged by alkylator-free regimen.

\section{Targeting Cathepsin S, a Regulator of Antigen Processing, to Enhance Immunogenicity of Lymphoma Cells}

\section{Highlighted by Riccardo Dolcetti}

The early stages of development of follicular lymphomas are characterized by the dependence of lymphoma cells from interactions with follicular T helper cells and follicular dendritic cells. Dheilly [23] and collaborators demonstrated that these pathogenic interactions are at least in part mediated by overexpression and recurrent activating mutations of Cathepsin S (CTSS). In addition to sustaining lymphoma cell survival and proliferation, the aberrant expression of CTSS was also shown to limit the ability of $\mathrm{CD}^{+} \mathrm{T}$ cells to recognize and eliminate lymphoma cells. Notably, inhibition of CTSS in preclinical models diversified the antigen repertoire of lymphoma cells, thereby promoting their recognition by cytotoxic $\mathrm{T}$ lymphocytes. These findings highlight the therapeutic relevance of strategies able to induce antigen diversification and indicate that targeting CTSS may improve adaptive immunity against lymphomas and responses to immunotherapies.

\section{Treatment for Glucocorticoid-Refractory Acute GVHD}

Highlighted by Marie Robin

Results of the REACH-2 trial have just been published in the New England Journal of Medicine by Zeiser et al. [24]. REACH-2 is an international multicenter, randomized, open-label, phase 3 trial comparing the efficacy and safety of oral ruxolitinib (10 $\mathrm{mg}$ twice daily) with other therapy commonly used (control) in patients with glucocorticoid-refractory acute GVHD after allogeneic stem-cell transplantation. The primary end point was overall response on day 28 . For the first time, a treatment, i.e., ruxolitinib, showed a significant improvement in the response rate (62\% vs. 39\%). Furthermore, the incidence of loss of response was lower with ruxolitinib at six months $(10 \% \mathrm{vs}$. $39 \%$ ). Non-relapse mortality was not significantly different between the two arms of treatment. Few randomized trials have been conducted in glucocorticoid-refractory GVHD and ruxolitinib is the only treatment showing a significant benefit in terms of sustained response. Ruxolitinib is the only treatment currently approved by FDA for this indication.

\section{Intermediate-1 Myelofibrosis also Benefits from Allogeneic Transplantation}

Highlighted by Rodrigo Martino

Although allogeneic hematopoietic stem cell transplantation (alloHCT) is the curative option in primary myelofibrosis (PMF), and secondary MF (SMF) [25], few patients are actually even considered candidates and the patients' risk groups are usually underestimated [26].

In ELN/EBMT 2015 guidelines for identifying optimal alloHCT candidates [27], most doubts about indication focused on the intermediate- 1 using the DIPSS classification. The current study by Gowin at al., however, supports proceeding to alloHCT in this specific scenario [28]. These authors compared the outcome of 551 alloHCT recipients from 2000 to 2014 with that of 1377 contemporary non-HCT patients. In brief, their finding in the intermediate- 1 group ( $40 \%$ of all patients) was that alloHCT was associated with improved long-term OS, but only after +1 year of follow-up. This study thus concludes that alloHCT benefits transplant-eligible patients with int-1 or higher-risk PMF/SMF using the DIPSS. The author of this editorial considers that alloHCT is even more justified in int-1 patients using DIPSS-Plus. Another provocative finding in this study is that prior therapy with ruxolitinb was associated with improved OS in both cohorts. 
13. The Role of Allogeneic Hematopoietic Stem Cell Transplantation (HSCT) and Post-Transplant FLT3 Inhibitors for AML Patients with FLT3-Internal Tandem Duplication (ITD)

Highlighted by Alessandro Busca

Approximately 25-30\% of patients with newly diagnosed acute myeloid leukemia (AML) present mutations in the FMS-like tyrosine kinase 3 (FLT3) gene, either by internal tandem duplication (FLT3-ITD) of the juxtamembrane domain or by a point mutation involving the tyrosine kinase domain (TKD). The FLT3-ITD mutation is a predictor of a higher relapse rate and worse survival. Allogeneic HSCT remains an effective treatment option for FLT3-mutated AML patients, however, a consistent number of targeted therapies has been developed over the most recent years and might be incorporated in the treatment of these patients. A position paper from the EBMT has been recently published summarizing indications of HSCT and the potential optimization of post-transplant maintenance with FLT3 inhibitors [29]. Based on this statement, indication for allogeneic HSCT is controversial in FLT3-ITD patients who belong to the European leukemia net (ELN) favorable risk group (for instance FLT3-ITD mutation with $<0.5$ allelic ratio and concomitant NPM1 mutation) and achieved minimal residual disease negativity; HSCT may be delayed until first relapse. Post-HSCT maintenance with FLT3 inhibitors for patients receiving an allogeneic HSCT for FLT3-ITD AML is strongly recommended: at the present, sorafenib may be considered the preferred option, however, the therapy duration and the role of other FLT3 inhibitors need to be defined.

\section{How Myeloma Cells Promote Adipogenesis}

\section{Highlighted by Michèle Sabbah}

The suppression of bone formation is a hallmark of multiple myeloma and understanding the mechanisms by which myeloma cells disturb the bone marrow is fundamental. Liu et al. [30] studied the effects of the interactions between myeloma cells and mesenchymal stem cells (MSCs) on osteoblastogenesis. Using single cell RNA-sequencing, an in vitro coculture system, and mouse models, the authors found that myeloma cells shift the differentiation of MSCs into adipocytes rather than osteoblasts. Mechanistic studies revealed that integrin $\alpha 4$ on myeloma cells activated the adhesion molecule VCAM1 on MSCs and inhibited ubiquitylation of PPAR $\gamma 2$ through PKC-MURF1 signaling. The resulting increase in PPAR $\gamma 2$ enhanced adipogenesis and suppressed osteoblastogenesis from MSCs. Therefore, counteracting $\alpha 4-$ VCAM1-MURF1-mediated adipogenesis from MSCs may be a promising strategy to heal myeloma-induced bone resorption.

\section{Anticoagulation in Patients with Active Cancer}

\section{Highlighted by Salvatore De Rosa}

The Caravaggio trial recently provided further evidence on the efficacy and safety of apixaban for the treatment of venous thromboembolism (VTE) in patients with cancer [31]. Hence, the current evidence outlook shows that DOACs are noninferior to dalteparin to prevent VTE recurrence with no increase in major bleeding [32]. It emerges that higher-risk clinically relevant non-major bleeding (CRNMB) is mostly associated with gastrointestinal cancers. This reinforced evidence should inform clinical management of cancer patients. Careful selection of anticoagulants based on clinical history, cancer type, concomitant medications, and bleeding risk can improve the quality of care. For instance, patients with brain tumors or acute leukemia were excluded from the Caravaggio but reassuring data come from the Hokusai VTE Cancer and the SELECT-D. In case of multiple concomitant treatments, it should be remembered that fewer drug interactions are expected with edoxaban. As residual use of warfarin is expected for cost-related issues; financial costs should not be the main decision driver in this setting. Furthermore, the apparent increase in DOAC-related costs is largely counterbalanced by the reduction in healthcare costs related to the management of bleeding events. 


\section{Megakaryocytes $\mathrm{Ca}^{2+}$ Flow Alteration in the Mutant CALR Type 1 Related Myeloproliferative Neoplasms Pathogenesis}

\section{Highlighted by Maurizio Martini}

Di Buduo CA and colleagues [33] have recently explained the molecular mechanisms by which CARL type 1 mutation leads to myeloproliferative neoplasms (MPN), inserting a keystone helps to understand the pathogenesis in this disease. Carrying out the in vitro cellular and molecular studies, the authors demonstrated that type 1 mutant CALR leaves the endoplasmic reticulum (ER) of megakaryocytes (MKs), losing its physiological binding with two other ER proteins, STIM and ERp57. Disengaged STIM proteins are now able to dimerize, translocate to plasma membrane, and, after binding to Orai and TRPC cellular membrane channel proteins, determine an extracellular $\mathrm{Ca}^{2+}$ entry. $\mathrm{Ca}^{2+}$ intra-MK increment plays a central role in regulating many cell processes, including cell proliferation and metabolism. This report also highlighted the fundamental part of MK in the myeloproliferative neoplasm pathogenesis, opening the way to new therapeutic approaches in the CARL mutant-related MPN treatment.

\section{Intestinal Microbiota Diversity Restoration as a Potential Way to Decrease Non-Relapse Mortality in Allo-HCT}

\section{Highlighted by Francesco Onida}

Based on a centralized analysis of a longitudinal serial fecal sampling from a very large patient population undergoing allogeneic $\mathrm{HCT}$, the multicenter intecontinental prospective study from Peled J.U. et al. [34] confirmed previously published single-center experiences reporting the negative impact of intestinal microbiota diversity loss on allogeneic HCT outcome. In particular, the association of lower intestinal diversity in the periengraftment period with inferior survival was shown to be determined by higher transplant-related and GvHD-related mortality, whereas no difference was observed in cumulative incidence of post-transplantation relapse. Indeed, lower microbiota diversity did not impact on survival in patients in whom ex vivo T-cell depletion was selected for GvHD prophylaxis strategy. Worth emphasizing is the study showed that also lower baseline microbiota diversity-i.e., based on pretransplantation samples-is associated with inferior post-transplant survival.

Even though not adjusted for antibiotics exposures, nor for diet and nonantibiotic drugs, the results of this study strongly advocate the implementation of future trials aimed at investigating intestinal microbiota diversity restoration as a potentially effective way to decrease allo-HCT-associated non-relapse mortality.

\section{Light-Chain PGNMID: A Genuine Monoclonal Gammopathy of Renal Significance}

Highlighted by Pierre Aucouturier

Proliferative glomerulonephritis with non-organized monoclonal immunoglobulin (Ig) deposition (PGNMID), is considered a "monoclonal gammopathy of renal significance" (MGRS) in spite of the usual absence of an identified B-cell clone. Reported PGNMID features "immune-complex like" mono-isotypic Ig deposits (mostly of the rare IgG3 subclass) that are restricted to the glomerulus. However, mono-isotypy does not mean monoclonality, which has obvious consequences on therapeutic decisions.

Recently, Nasr et al. [35] reported on a well-documented series of 17 patients with atypical PGNMID featuring deposition of monoclonal Ig light chains (LC) only. Unlike classical IgG-PGNMID, a monoclonal gammopathy was identified in virtually all of them, including myeloma in one-third. Moreover, proteomic analyses of microdissected glomeruli demonstrated the monoclonal origin (homogenous variable domain sequences) of deposited material, which to my knowledge has not been demonstrated in classical IgG-PGNMID, contrary to other MGRS including light chain deposition disease [36]. Molecular studies also suggested activation of the complement alternative pathway. Thus, while treatments of IgG-PGNMID empirically target a hypothetical B-cell clone, LC-PGNMID appears as a distinct entity with a true rationale for the use of myeloma regimens. 


\section{Is Tandem Transplantation the Standard of Care?}

Highlighted by Fredrik Schjesvold

The EMN group is capable of running large randomized trials, asking several questions in one clinical study. EMN02 is such a study, asking whether transplant is good (yes), whether consolidation is good (probably), but more importantly, providing the best data to date on whether tandem ASCT is beneficial [37]. To be short, it is. In the ITT population with a $5 \mathrm{y}$ follow-up, overall survival was $80.3 \%$ in the tandem arm, $72.6 \%$ in the single ASCT arm, and 71.5\% in the group randomized to VMP instead of transplant. The OS benefit for tandem over single ASCT was statistically significant with an HR of 0.62, most prominently demonstrated in the HR group. At ASCO 2020, long-term follow-up from the STaMINA trial was presented, confirming PFS benefit of tandem ASCT, in the patients that received the allocated treatment (30\% did not). Further, in this study the results were more evident in the high-risk group. Based on this, the coming ESMO guidelines recommends tandem ASCT in HR patients, and it should be a definite option in the standard-risk patients as well.

\section{Chromatin Organization Contributes to T Cell Acute Lymphoblastic Leukemia Pathogenesis}

\section{Highlighted by Stéphane Minvielle}

Transcriptional regulation in mammalian cells is orchestrated by cis-regulatory elements that include promoters, enhancers, and insulators. A majority of them display cell-type specific chromatin accessibility and act as enhancers to regulate specific gene expression. Enhancers are often located at a great genomic distance from their target gene promoters, therefore correct assignment of enhancers to genes requires knowledge of spatial genome organization. Chromatin structure changes and their impact in tumor development remain largely unknown. In the present study, Kloetgen et al. in 2019 filled this gap [38]. The authors explored the potential reorganization of a distinct chromatin structure called TAD in primary T-ALL compared to T cells. They identified significant alterations in intra-TAD chromatin interactions affecting important genes for T-ALL pathogenesis including NOTCH1 targets genes as well as TAD boundary changes including a TAD fusion that conferred spatial proximity of MYC promoter and the super-enhancer. They revealed the importance of NOTCH1 binding activity that maintains specific enhancer-promoter loops. Overall this study opens the way to identify and target factors like NOTCH1 that orchestrate the interactions between super-enhancers and gene promoters in cancer cells.

\section{The Thrombotic Storm in COVID-19 Pandemic Outbreak: The Link between Inflammation and Haemostasis}

\section{Highlighted by Mario Mazzucato}

The coronavirus disease 2019 (COVID-19) pandemic has besieged us with its relentless worldwide march and high morbidity and mortality [39]. COVID-19 appears to preferentially target respiratory epithelium where it enters host cells through the angiotensin converting enzyme 2 receptor, similar to SARS-CoV.

The triggering of host inflammatory reactions also results in increased production of proinflammatory cytokines that have pleiotropic effects, including activation of coagulation. Factor XII (FXII) is the zymogen of serine protease, factor XIIa (FXIIa). In vivo, FXIIa initiates coagulation via the fibrin-forming "intrinsic" pathway and promotes inflammation via the bradykinin (BK)-producing kallikrein kinin system, comprising high-molecular weight kininogen $(\mathrm{HK})$ and plasma prekallikrein (PK) [40]. Together, FXII, PK, and HK are termed the plasma contact activation system. Because of its original identification as part of the coagulation intrinsic pathway, the inflammatory branch of the contact activation system has long been underappreciated. In addition to neutrophil pro-inflammatory responses, FXII modulates the functions of an array of innate immune cells. Macrophages react to FXII 
and FXIIa by increasing the production of IL-6, IL-12, and tumor necrosis factor- $\alpha$. Finally, FXII was shown to promote the differentiation of T helper naive cells to TH17 cells [41].

Although not considered part of the contact system in vitro, plasmin has a known capacity for FXII activation. In addition, recent studies indicate that soluble polyP enhances the plasminogen activator function of FXIIa (hyperfibrinolytic states).

A rapid global community effort has been made to integrate new clinic and laboratory observation, involving coagulation and fibrinolytic pathways, to help guide patient care with COVID-19. Studying the intrinsic pathway of coagulation in COVID-19 may be the true keystone to understanding thrombotic complications. Our understanding of this new human pathogen is rapidly evolving, and our approach to patient management must continue to evolve.

\section{We Are History: Studying the Present to Understand the Past of Multiple Myeloma (MM)}

\section{Highlighted by Carolina Terragna}

The study of Rustad E.A. et al. [42] shows that several mutational processes actively shape the genome of MM patients, starting years before the onset of the clinical symptoms. Consequently, the genomic snapshot provided by the bone marrow aspirates commonly obtained before therapy allows a proper risk stratification of patients, according to the presence of structural and numerical chromosomal aberrations. More importantly, it also tells about the patients' tumor history, providing one can read the genomic footprints, which remind of individual habits, aging, exposure to genotossic agents, and random activation of (eventually) oncogenic pathways occurring throughout the whole lifetime.

These so-called mutational signatures mark any given DNA sample and can be read by high-throughput technologies, allowing to trespass the outer surface of chromosomes, by deepening into their biochemical information core, made of nucleotides liable to mutations [43].

With an MM genomic picture at the onset of the disease being the result of a long and progressive genomic ride, intercepting the MM evolutive process might delay or even prevent the disease progression, thus supporting the possibility to therapeutically change the natural history of this disease [44].

\section{On the Road to Newer and Safer CARs}

Highlighted by Michel Delforge

CAR-T cells are reshaping the treatment paradigm of patients with relapsed and refractory lymphoproliferative disorders. Currently anti-CD19 CAR T-cells are licensed for the treatment of subgroups of patients with relapsed/refractory diffuse large B-cell lymphoma and refractory ALL. In multiple myeloma, several phase II studies have shown impressive results with CAR-T cells directed towards BCMA. However, despite impressive response rates, many patients will relapse after CAR-T treatment. Moreover, the infusion of CAR-T cells can be complicated by cytokine release syndrome (CRS) and neurotoxicity. Therefore more efforts are needed to improve efficacy and reduce toxicities of genetically engineered cell therapies. Another limitation is the autologous source requiring several weeks of manufacturing time and the use of autologous cells from patients who have been exposed to several immunosuppressive drugs. A potential strategy to overcome these drawbacks is the use of allogeneic NK cells [45]. Despite being a small phase 1/2 study, it supports the proof-of-concept of using CAR-NK cells from cord blood as an effective treatment without causing major toxicities and may be a potential future alternative to CAR T-cells.

\section{Erythocyte Microvesicles and Thrombosis in MPN}

\section{Highlighted by Claire Harrison}

The dominant clinical feature of the Philadelphia negative myeloproliferative neoplasms (MPN) is vascular events. Some $30 \%$ of patients will have a vascular event (mostly arterial) at or within a few 
years of diagnosis. The cause of thrombosis and its relationship with the commonest driver mutation, $J A K 2 \mathrm{~V} 617 \mathrm{~F}$, is unclear. In this paper, Poisson and colleagues [46] reveal a putative mechanism involving JAK2 V617F erythrocyte-derived microvesicles carrying MPO, and that these are responsible for increased oxidative stress in arterial endothelium and decreased availability of $\mathrm{NO}$, which strongly increased arterial contraction in response to vasoconstrictive agents, possibly accounting for the arterial events associated with MPNs. This was demonstrated with a series of mouse models and could explain in part the features of coronary events without coronary artery disease in these patients. This paper also highlights the potential role of NO pathway inhibition and increased endothelial oxidative stress in arterial constriction in MPN. Several groups have reported high levels of circulating ROS products [47-49] and low antioxidant status in MPN [48,50]. Erythrocyte microvesicles have already been linked to vascular dysfunction in various settings, such as erythrocyte storage and sickle cell disease $[47,48,51]$, but not in the context of MPN. Using proteomics, a defect in GSTT1 and overexpression of MPO in microvesicles derived from JAK2 V617F erythrocytes link this to oxidative stress. This opens new therapeutic perspectives and the authors demonstrated the benefits of simvastatin and hydroxycarbamide.

Conflicts of Interest: The authors declare no conflict of interest.

\section{References}

1. Wright, G.W.; Huang, D.W.; Phelan, J.D.; Coulibaly, Z.A.; Roulland, S.; Young, R.M.; Wang, J.Q.; Schmitz, R.; Morin, R.D.; Tang, J.; et al. A Probabilistic Classification Tool for Genetic Subtypes of Diffuse Large B Cell Lymphoma with Therapeutic Implications. Cancer Cell 2020, 37, 551-568.e14. [CrossRef] [PubMed]

2. Schmitz, R.; Wright, G.W.; Huang, D.W.; Johnson, C.A.; Phelan, J.D.; Wang, J.Q.; Roulland, S.; Kasbekar, M.; Young, R.M.; Shaffer, A.L.; et al. Genetics and Pathogenesis of Diffuse Large B-Cell Lymphoma. N. Engl. J. Med. 2018, 378, 1396-1407. [CrossRef] [PubMed]

3. Chapuy, B.; Stewart, C.; Dunford, A.J.; Kim, J.; Kamburov, A.; Redd, R.A.; Lawrence, M.S.; Roemer, M.G.M.; Li, A.J.; Ziepert, M.; et al. Molecular subtypes of diffuse large B cell lymphoma are associated with distinct pathogenic mechanisms and outcomes. Nat. Med. 2018, 24, 679-690. [CrossRef] [PubMed]

4. Lacy, S.E.; Barrans, S.L.; Beer, P.A.; Painter, D.; Smith, A.G.; Roman, E.; Cooke, S.L.; Ruiz, C.; Glover, P.; Van Hoppe, S.J.L.; et al. Targeted sequencing in DLBCL, molecular subtypes, and outcomes: A Haematological Malignancy Research Network report. Blood 2020, 135, 1759-1771. [CrossRef] [PubMed]

5. Angelucci, E.; Li, J.; Greenberg, P.; Wu, D.; Hou, M.; Figueroa, E.H.M.; Rodriguez, M.G.; Dong, X.; Ghosh, J.; Izquierdo, M.; et al. Iron Chelation in Transfusion-Dependent Patients With Low- to Intermediate-1-Risk Myelodysplastic Syndromes. Ann. Intern. Med. 2020, 172, 513-522. [CrossRef]

6. Drayson, M.T.; Bowcock, S.; Planche, T.; Iqbal, G.; Pratt, G.; Yong, K.; Wood, J.; Raynes, K.; Higgins, H.; Dawkins, B.; et al. Levofloxacin prophylaxis in patients with newly diagnosed myeloma (TEAMM): A multicentre, double-blind, placebo-controlled, randomised, phase 3 trial. Lancet Oncol. 2019, 20, 1760-1772. [CrossRef]

7. Zavidij, O.; Haradhvala, N.J.; Mouhieddine, T.H.; Sklavenitis-Pistofidis, R.; Cai, S.; Reidy, M.; Rahmat, M.; Flaifel, A.; Ferland, B.; Su, N.K.; et al. Single-cell RNA sequencing reveals compromised immune microenvironment in precursor stages of multiple myeloma. Nat. Rev. Cancer 2020, 1, 493-506. [CrossRef]

8. De Magalhães, R.J.P.; Vidriales, M.-B.; Paiva, B.; Fernandez-Gimenez, C.; García-Sanz, R.; Mateos, M.-V.; Gutierrez, N.C.; Lecrevisse, Q.; Blanco, J.F.; Hernández, J.; et al. Analysis of the immune system of multiple myeloma patients achieving long-term disease control by multidimensional flow cytometry. Haematologica 2012, 98, 79-86. [CrossRef]

9. Bustoros, M.; Sklavenitis-Pistofidis, R.; Park, J.; Redd, R.; Zhitomirsky, B.; Dunford, A.J.; Salem, K.; Tai, Y.-T.; Anand, S.; Mouhieddine, T.H.; et al. Genomic Profiling of Smoldering Multiple Myeloma Identifies Patients at a High Risk of Disease Progression. J. Clin. Oncol. 2020. [CrossRef]

10. Thurner, L.; Hartmann, S.; Fadle, N.; Regitz, E.; Kemele, M.; Kim, Y.-J.; Bohle, R.M.; Nimmesgern, A.; Von Müller, L.; Kempf, V.A.J.; et al. Lymphocyte predominant cells detect Moraxella catarrhalis-derived antigens in nodular lymphocyte-predominant Hodgkin lymphoma. Nat. Commun. 2020, 11, 1-12. [CrossRef] 
11. Prakash, S.; Fountaine, T.; Raffeld, M.; Jaffe, E.S.; Pittaluga, S. IgD Positive L\&H Cells Identify a Unique Subset of Nodular Lymphocyte Predominant Hodgkin Lymphoma. Am. J. Surg. Pathol. 2006, 30, 585-592. [CrossRef] [PubMed]

12. Stray-Gundersen, J.; Chapman, R.; Levine, B.D. "Living high-training low" altitude training improves sea level performance in male and female elite runners. J. Appl. Physiol. 2001, 91, 1113-1120. [CrossRef] [PubMed]

13. Staub, K.; Haeusler, M.; Bender, N.; Morozova, I.; Eppenberger, P.; Panczak, R.; Zwahlen, M.; Schaer, D.J.; Maggiorini, M.; Ulrich, S.; et al. Hemoglobin concentration of young men at residential altitudes between 200 and 2000 m mirrors Switzerland's topography. Blood 2020, 135, 1066-1069.

14. Drakesmith, H.; Prentice, A.M. Viral infection and iron metabolism. Nat. Rev. Genet. 2008, 6, 541-552. [CrossRef]

15. Litton, E.; Lim, J. Iron Metabolism: An Emerging Therapeutic Target in Critical Illness. Crit. Care 2019, 23, 81. [CrossRef]

16. Wu, C.; Chen, X.; Cai, Y.; Xia, J.; Zhou, X.; Xu, S.; Huang, H.; Zhang, L.; Zhou, X.; Du, C.; et al. Risk Factors Associated With Acute Respiratory Distress Syndrome and Death in Patients With Coronavirus Disease 2019 Pneumonia in Wuhan, China. JAMA Intern. Med. 2020, 13, 200994. [CrossRef]

17. Shah, A.; Frost, J.; Aaron, L.; Donovan, K.; McKechnie, S.; Stanworth, S.; Drakesmith, H. Systemic hypoferraemia and severity of hypoxaemic respiratory failure in COVID-19. Crit. Care 2020, 24, 320. [CrossRef]

18. National Comprehensive Cancer Network. NCCN Clinical Practice Guidelines in Oncology: Multiple Myeloma Version 2. 2019. Available online: https://www.nccn.org/professionals/physician_gls/pdf/myeloma. pdf (accessed on 3 September 2019).

19. Mateos, M.-V.; Cavo, M.; Blade, J.; Dimopoulos, M.A.; Suzuki, K.; Jakubowiak, A.; Knop, S.; Doyen, C.; Lucio, P.; Nagy, Z.; et al. Overall survival with daratumumab, bortezomib, melphalan, and prednisone in newly diagnosed multiple myeloma (ALCYONE): A randomised, open-label, phase 3 trial. Lancet 2020, 395, 132-141. [CrossRef]

20. Krejcik, J.; Casneuf, T.; Nijhof, I.S.; Verbist, B.; Bald, J.; Plesner, T.; Syed, K.; Liu, K.; Van De Donk, N.W.C.J.; Weiss, B.M.; et al. Daratumumab depletes CD38+ immune regulatory cells, promotes T-cell expansion, and skews T-cell repertoire in multiple myeloma. Blood 2016, 128, 384-394. [CrossRef]

21. Facon, T.; Kumar, S.; Plesner, T.; Orlowski, R.Z.; Moreau, P.; Bahlis, N.; Basu, S.; Nahi, H.; Hulin, C.; Quach, H.; et al. Daratumumab plus Lenalidomide and Dexamethasone for Untreated Myeloma. N. Engl. J. Med. 2019, 380, 2104-2115. [CrossRef]

22. ClinicalTrials.gov, NCT03652064. Available online: https://clinicaltrials.gov/ct2/show/NCT03652064 (accessed on 24 June 2020).

23. Dheilly, E.; Battistello, E.; Katanayeva, N.; Sungalee, S.; Michaux, J.; Duns, G.; Wehrle, S.; Sordet-Dessimoz, J.; Mina, M.; Racle, J.; et al. Cathepsin S Regulates Antigen Processing and T Cell Activity in Non-Hodgkin Lymphoma. Cancer Cell 2020, 37, 674-689. [CrossRef] [PubMed]

24. Zeiser, R.; Von Bubnoff, N.; Butler, J.; Mohty, M.; Niederwieser, D.; Or, R.; Szer, J.; Wagner, E.M.; Zuckerman, T.; Mahuzier, B.; et al. Ruxolitinib for Glucocorticoid-Refractory Acute Graft-versus-Host Disease. N. Engl. J. Med. 2020, 382, 1800-1810. [CrossRef] [PubMed]

25. Tefferi, A. Management of Primary Myelofibrosis. Available online: https://www.uptodate.com/contents/ management-of-primary-myelofibrosis (accessed on 1 May 2020).

26. Verstovsek, S.; Yu, J.; Kish, J.K.; Paranagama, D.; Kaufman, J.; Myerscough, C.; Grunwald, M.R.; Colucci, P.; Mesa, R. Real-world risk assessment and treatment initiation among patients with myelofibrosis at community oncology practices in the United States. Ann. Hematol. 2020, 20, 1-10. [CrossRef]

27. Kröger, N.M.; Deeg, J.H.; Olavarria, E.; Niederwieser, D.; Bacigalupo, A.; Barbui, T.; Rambaldi, A.; Mesa, R.; Tefferi, A.; Griesshammer, M.; et al. Indication and management of allogeneic stem cell transplantation in primary myelofibrosis: A consensus process by an EBMT/ELN international working group. Leukemia 2015, 29, 2126-2133. [CrossRef] [PubMed]

28. Gowin, K.; Ballen, K.; Ahn, K.W.; Hu, Z.-H.; Ali, H.; Arcasoy, M.O.; Devlin, R.; Coakley, M.; Gerds, A.T.; Green, M.; et al. Survival following allogeneic transplant in patients with myelofibrosis. Blood Adv. 2020, 4, 1965-1973. [CrossRef] [PubMed] 
29. Bazarbachi, A.; Bug, G.; Baron, F.; Brissot, E.; Ciceri, F.; Dalle, I.A.; Döhner, H.; Esteve, J.; Floisand, Y.; Giebel, S.; et al. Clinical practice recommendation on hematopoietic stem cell transplantation for acute myeloid leukemia patients with FLT3-internal tandem duplication: A position statement from the Acute Leukemia Working Party of the European Society for Blood and Marrow Transplantation. Haematologica 2020, 105, 1507-1516. [CrossRef]

30. Liu, Z.; Liu, H.; He, J.; Lin, P.; Tong, Q.; Yang, J. Myeloma cells shift osteoblastogenesis to adipogenesis by inhibiting the ubiquitin ligase MURF1 in mesenchymal stem cells. Sci. Signal. 2020, 13, 633. [CrossRef]

31. Agnelli, G.; Becattini, C.; Meyer, G.; Muñoz, A.; Huisman, M.V.; Connors, J.M.; Cohen, A.; Bauersachs, R.; Brenner, B.; Torbicki, A.; et al. Apixaban for the Treatment of Venous Thromboembolism Associated with Cancer. N. Engl. J. Med. 2020, 382, 1599-1607. [CrossRef]

32. Sabatino, J.; De Rosa, S.; Polimeni, A.; Sorrentino, S.; Indolfi, C. Direct Oral Anticoagulants (DOACs) in Patients with Active Cancer: A Systematic Review and Meta-Analysis. JACC CardioOncol. 2020, pii:S2666-0873(20)30149-6, in press.

33. Di Buduo, C.A.; Abbonante, V.; Marty, C.; Moccia, F.; Rumi, E.; Pietra, D.; Soprano, P.M.; Lim, D.; Cattaneo, D.; Iurlo, A.; et al. Defective interaction of mutant calreticulin and SOCE in megakaryocytes from patients with myeloproliferative neoplasms. Blood 2020, 135, 133-144. [CrossRef]

34. Peled, J.U.; Gomes, A.; Devlin, S.M.; Littmann, E.R.; Taur, Y.; Sung, A.D.; Weber, D.; Hashimoto, D.; Slingerland, A.E.; Slingerland, J.B.; et al. Microbiota as Predictor of Mortality in Allogeneic Hematopoietic-Cell Transplantation. N. Engl. J. Med. 2020, 382, 822-834. [CrossRef]

35. Nasr, S.H.; Larsen, C.P.; Sirac, C.; Theis, J.D.; Domenger, C.; Chauvet, S.; Javaugue, V.; Hogan, J.J.; Said, S.M.; Dasari, S.; et al. Light chain only variant of proliferative glomerulonephritis with monoclonal immunoglobulin deposits is associated with a high detection rate of the pathogenic plasma cell clone. Kidney Int. 2020, 97, 589-601. [CrossRef] [PubMed]

36. Cogné, M.; Preud'Homme, J.L.; Bauwens, M.; Touchard, G.; Aucouturier, P. Structure of a monoclonal kappa chain of the V kappa IV subgroup in the kidney and plasma cells in light chain deposition disease. J. Clin. Investig. 1991, 87, 2186-2190. [CrossRef] [PubMed]

37. Cavo, M.; Gay, F.; Beksac, M.; Pantani, L.; Petrucci, M.T.; Dimopoulos, M.A.; Dozza, L.; Van Der Holt, B.; Zweegman, S.; Oliva, S.; et al. Autologous haematopoietic stem-cell transplantation versus bortezomib-melphalan-prednisone, with or without bortezomib-lenalidomide-dexamethasone consolidation therapy, and lenalidomide maintenance for newly diagnosed multiple myeloma (EMN02/HO95): A multicentre, randomised, open-label, phase 3 study. Lancet Haematol. 2020, 7, e456-e468. [CrossRef]

38. Kloetgen, A.; Thandapani, P.; Ntziachristos, P.; Ghebrechristos, Y.; Nomikou, S.; Lazaris, C.; Chen, X.; Hu, H.; Bakogianni, S.; Wang, J.; et al. Three-dimensional chromatin landscapes in T cell acute lymphoblastic leukemia. Nat. Genet. 2020, 52, 388-400. [CrossRef]

39. Connors, J.M.; Levy, J.H. COVID-19 and its implications for thrombosis and anticoagulation. Blood 2020, 135, 2033-2040. [CrossRef]

40. Renné, T.; Schuh, K.; Müller-Esterl, W. Local bradykinin formation is controlled by glycosaminoglycans. J. Immunol. 2005, 175, 3377-3385. [CrossRef] [PubMed]

41. Manthey, H.D.; Cochain, C.; Busch, M.; Chaudhari, S.M.; Stegner, D.; Yepes, M.; Lorenz, K.; Nolte, M.W.; Nieswandt, B.; Vorlova, S.; et al. Coagulation factor XII induces pro-inflammatory cytokine responses in macrophages and promotes atherosclerosis in mice. Thromb. Haemost. 2017, 117, 176-187. [CrossRef]

42. Rustad, E.H.; Yellapantula, V.; Leongamornlert, D.; Bolli, N.; Ledergor, G.; Nadeu, F.; Angelopoulos, N.; Dawson, K.J.; Mitchell, T.J.; Osborne, R.J.; et al. Timing the initiation of Multiple Myeloma. Nat. Commun. 2020, 11, 1917. [CrossRef]

43. Gerstung, M.; PCAWG Evolution \& Heterogeneity Working Group; Jolly, C.; Leshchiner, I.; Dentro, S.C.; Gonzalez, S.; Rosebrock, D.; Mitchell, T.J.; Rubanova, Y.; Anur, P.; et al. The evolutionary history of 2,658 cancers. Nature 2020, 578, 122-128. [CrossRef] [PubMed]

44. Lomas, O.C.; Ghobrial, I.M. Clinical controversies in the management of Smoldering Multiple Myeloma. Am. Soc. Clin. Oncol. Educ. Book 2020, 40, 1-6. [CrossRef] [PubMed]

45. Liu, E.; Marin, D.; Banerjee, P.; Macapinlac, H.; Thompson, P.; Basar, R.; Kerbauy, L.; Overman, B.; Thall, P.; Kaplan, M.; et al. Use of CAR-Transduced Natural Killer Cells in CD19-Positive Lymphoid Tumors. N. Engl. J. Med. 2020, 382, 545-553. [CrossRef] 
46. Poisson, J.; Tanguy, M.; Davy, H.; Camara, F.; Mdawar, M.-B.E.; Kheloufi, M.; Dagher, T. Devue, C.; Lasselin, J.; Plessier, A.; et al. Erythrocyte-derived microvesicles induce arterial spasms in JAK2V617F myeloproliferative neoplasm. J Clin Invest. 2020, 130, 2630-2643. [CrossRef] [PubMed]

47. Donadee, C.; Raat, N.J.H.; Kanias, T.; Tejero, J.; Lee, J.S.; Kelley, E.E.; Zhao, X.; Liu, C.; Reynolds, H.; Azarov, I.; et al. Nitric oxide scavenging by red blood cell microparticles and cell-free hemoglobin as a mechanism for the red cell storage lesion. Circulation. 2011, 124, 465-476. [CrossRef] [PubMed]

48. Camus, S.M.; De Moraes, J.A.; Bonnin, P.; Abbyad, P.; Le Jeune, S.; Lionnet, F.; Loufrani, L.; Grimaud, L.; Lambry, J.-C.; Charue, D.; et al. Circulating cell membrane microparticles transfer heme to endothelial cells and trigger vasoocclusions in sickle cell disease. Blood 2015, 125, 3805-3814. [CrossRef] [PubMed]

49. Vanhoutte, P.M.; Zhao, Y.; Xu, A.; Leung, S.W. Thirty years of saying NO: Sources, fate, actions, and misfortunes of the endothelium-derived vasodilator mediator. Circ. Res. 2016, 119, 375-396. [CrossRef]

50. Stocker, R.; Keaney, J.F. Role of oxidative modifications in atherosclerosis. Physiol Rev. 2004, 84, 1381-1478. [CrossRef] [PubMed]

51. Said, A.S.; Doctor, A. Influence of red blood cell-derived microparticles upon vasoregulation. Blood Transfus. 2017, 15, 522-534.

(C) 2020 by the authors. Licensee MDPI, Basel, Switzerland. This article is an open access article distributed under the terms and conditions of the Creative Commons Attribution (CC BY) license (http://creativecommons.org/licenses/by/4.0/). 